\section{Schönheimer, Rudolf}

H. Fiedler

Erfurt, Deutschland

Lebensdaten Deutsch-amerikanischer Mediziner und Biochemiker, geboren 1898 in Berlin, gestorben 1941 in Yonkers (USA). Nach der Promotion forschte Schönheimer (auch Schoenheimer) am Moabit-Krankenhaus über Steroide als Risikofaktoren der Atherosklerose. Nach Weiterbildung in Biochemie bei Karl Thomas in Leipzig leitete er 1926 in Freiburg das Chemische Laboratorium des Pathologischen Instituts von Ludwig Aschoff (1876-1942). Hier hatte er wichtige Kontakte zu Adolf Windaus ( $\triangleright$ Windaus, Adolf Otto Reinhold), Georg von Hevesy (Tracertechnik, Neutronenaktivierungsanalyse), Sir Hans Adolf Krebs (Zitrat- und Harnstoffzyklus) und Siegfried Thannhauser (Stoffwechselforschung). 1933 musste er als Jude zusammen mit seiner jüdischen Frau (Salome Gluecksohn-Waelsch, 1907-2007, Begründerin der Entwicklungsgenetik der Säugetiere) Freiburg verlassen und ging zu Hans T. Clark, Department of Biological Chemistry, Columbia College New York.
Verdienste Bereits in Deutschland hatte Schönheimer erkannt, dass der intermediäre Stoffwechsel einer schnellen Dynamik unterliegt. Wichtiges Hilfsmittel war derzeit die Markierung von Substanzen: Phenylsubstituierung von Fettsäuren durch F. Knoop, Isotopenmarkierung durch von Hevesy und Einführung des Massenspektrografen durch F.W. Aston. In Amerika konnte Schönheimer die Isolierung von Deuterium/schwerem Wasser (1934) und von ${ }^{15} \mathrm{~N}$ (1937) durch H.C. Urey nutzen. Zusammen mit D. Rittenberg, Sarah Ratner und Konrad E. Bloch führte Schönheimer zahlreiche Turnover-Untersuchungen durch: Stoffwechselwege diätetisch zugeführter Fettsäuren, Bestätigung des Harnstoffzyklus, Biosynthese von Cholesterin, Kreatin, Hippursäure sowie von Aminosäuren und deren Transaminierung. Schönheimer wird als Vater der Molekularen Biochemie betrachtet. Sein selbst gewählter früher Tod hat wohl die Auszeichnung mit dem Nobelpreis verhindert.

\section{Literatur}

Berthold HK (2003) Rudolf Schönheimer (1898-1941): Leben und Werk, 2. Aufl. Falk Foundation, Freiburg i. Br

Guggenheim KY (1991) Rudolf Schoenheimer and the concept of the dynamic state of body constituents. J Nutr 121:1701-1704 